ThE ASTRophysical Journal, 264:L69-L70, 1983 January 15

(C) 1983. The American Astronomical Society. All rights reserved. Printed in U.S.A.

\title{
LABORATORY MILLIMETER AND SUBMILLIMETER SPECTRUM OF HOC ${ }^{+}$
}

\author{
Geoffrey A. BlaKe \\ Department of Chemistry, California Institute of Technology \\ Paul Helminger \\ Department of Physics, University of South Alabama \\ AND \\ Eric Herbst and Frank C. De Lucia \\ Department of Physics, Duke University \\ Received 1982 August 18; accepted 1982 September 21
}

\begin{abstract}
The $J=1 \rightarrow 2,2 \rightarrow 3$, and $3 \rightarrow 4$ rotational transitions of the molecular ion $\mathrm{HOC}^{+}$have been measured in the laboratory at frequencies from 178 to $358 \mathrm{GHz}$. Our data should permit astronomers to confirm the recent possible sighting of the $J=1 \rightarrow 0$ transition of $\mathrm{HOC}^{+}$in $\mathrm{Sgr} \mathrm{B} 2$ at $89.5 \mathrm{GHz}$. Subject headings: interstellar: molecules - laboratory spectra
\end{abstract}

Based on the laboratory detection of $\mathrm{HOC}^{+}$by Gudeman and Woods (1982), Woods et al. (1982) have recently observed and tentatively identified a weak emission line in $\operatorname{Sgr} \mathrm{B} 2$ as the $J=1 \rightarrow 0$ transition of $\mathrm{HOC}^{+}$. Assuming the correctness of the identification, Woods et al. (1982) obtained an $\mathrm{HCO}^{+} / \mathrm{HOC}^{+}$abundance ratio of $\sim 330$ and used a model consisting of $\sim 20$ ion-molecule reactions to deduce that the large abundance ratio implies the existence of a significant abundance of $\mathrm{H} \mathrm{I}$ in dense interstellar clouds. In this Letter, we report the laboratory measurement of three additional rotational transitions of $\mathrm{HOC}^{+}$. These transitions, with rotational quantum numbers $J=1 \rightarrow 2,2$ $\rightarrow 3$, and $3 \rightarrow 4$, lie in the millimeter and submillimeter regions of the electromagnetic spectrum and help confirm the laboratory identification of $\mathrm{HOC}^{+}$. The $J=2$ $\rightarrow 3$ and $3 \rightarrow 4$ lines, at 268.5 and $357.9 \mathrm{GHz}$, respectively, should be observable by astronomers. Interstellar observation of $\mathrm{HOC}^{+}$at higher frequencies would confirm the identification of this species in Sgr B2 by Woods et al. (1982).

Our millimeter and submillimeter spectroscopic techniques have been discussed previously (De Lucia 1976). The $\mathrm{HOC}^{+}$ion was produced in a 5 foot long, 1.5 inch diameter discharge cell at liquid $\mathrm{N}_{2}$ temperature that was covered by a solenoid to produce axial magnetic fields of up to 200 gauss. A discharge current of $10 \mathrm{~mA}$ was maintained at a voltage of $5 \mathrm{kV}$. The total pressure in the cell was 10 mtorr, and the chief constituent was argon, with trace amounts of $\mathrm{H}_{2}$ and $\mathrm{CO}$ (Gudeman and Woods 1982). The magnetic field enhanced the signals sufficiently to permit detection of $\mathrm{HOC}^{+}$with lock-in signal recovery at a time constant of $1 \mathrm{~s}$. We have previously discussed the large enhancement in sig- nal attainable via magnetic confinement of molecular ions such as $\mathrm{NO}^{+}, \mathrm{HCO}^{+}$, etc. (Bowman, Herbst, and De Lucia 1982).

The transition frequencies measured in this experiment are listed in Table 1. These frequencies have been corrected for a small but unusual Doppler shift in the following manner. The $J=2 \rightarrow 3$ transition was observed with both relative directions of the microwave beam and the ion drift velocity by placing the detector at different ends of the cell. It was found that the net direction of ion flow was opposite to the sense expected from the polarity of the electrodes. Presumably, this effect was caused by the vacuum pump operating on a small-diameter discharge tube.

The rotational constants $B_{0}$ and $D_{0}$ obtained from a least squares fit to the data are also listed in Table 1. These constants permit accurate calculation of rest frequencies for a wide range of transitions. For the $J=0$ $\rightarrow 1$ transition, we predict a frequency of $89,487.387(28)$ $\mathrm{MHz}$, in good agreement with the value of $89,487.414(15)$

TABLE 1

Measured Rotational Transitions OF $\mathrm{HOC}^{+}(\mathrm{MHz})$

\begin{tabular}{ccc}
\hline \hline $\begin{array}{c}\text { Transition } \\
J^{\prime \prime} \rightarrow J^{\prime}\end{array}$ & Observed $^{\mathrm{a}}$ & Obs. - Calc. $^{\mathrm{b}}$ \\
\hline $1 \rightarrow 2 \ldots \ldots \ldots$ & 178972.051 & 0.034 \\
$2 \rightarrow 3 \ldots \ldots \ldots$ & 268451.094 & -0.038 \\
$3 \rightarrow 4 \ldots \ldots \ldots$ & 357921.987 & 0.012 \\
\hline
\end{tabular}

${ }^{a}$ Includes shift to cancel Doppler effect caused by ion drift velocity and vacuum pump.

${ }^{b}$ Fitted constants are $B_{0}=44,743.9235(140)$ $\mathrm{MHz}$ and $D_{0}=114.89(54) \mathrm{kHz}$. 


\section{BLAKE, HELMINGER, HERBST, AND DE LUCIA}

phase space theory of reaction collisions (Light 1967)

$\mathrm{MHz}$ obtained by Gudeman and Woods (1982). As 'ol' expected (Gudeman and Woods 1982), the centrifugal $1-1$ distortion constant, $D_{0}$, of $\mathrm{HOC}^{+}$is significantly larger than that of $\mathrm{HCO}^{+}$.

The analysis of the observed $\mathrm{HCO}^{+} / \mathrm{HOC}^{+}$abundance ratio in Sgr B2 by Woods et al. (1982) leads to the conclusion that the relative weakness of the signal ascribed to $\mathrm{HOC}^{+}$is due primarily to the selective depletion reaction, $\mathrm{HOC}^{+}+\mathrm{H} \rightarrow \mathrm{H}+\mathrm{OCH}^{+}$An atomic hydrogen abundance considerably greater than the $\mathrm{CO}$ abundance appears to be required, which is in conflict with ion-molecule model predictions. Basic to the analysis is the assumption that the reaction between $\mathrm{CO}$ and $\mathrm{H}_{3}^{+}$produces equal amounts of $\mathrm{HCO}^{+}$and $\mathrm{HOC}^{+}$. While this may be true, it is in conflict with the which predicts the more exothermic pathway $\left(\mathrm{HCO}^{+}+\right.$ $\mathrm{H}_{2}$ ) to be dominant. In addition, recent experimental work by Illies, Jarrold, and Bowers (1982) on collisioninduced dissociation patterns indicates that the reaction between $\mathrm{H}_{3}^{+}$and $\mathrm{CO}$ produces between $2 \%$ and $10 \%$ $\mathrm{HOC}^{+}+\mathrm{H}_{2}$. If we utilize the $2 \%$ figure, then an $\mathrm{HCO}^{+} / \mathrm{HOC}^{+}$abundance ratio of $\sim 50$ can be caused by the difference in production rates, and the abundance of atomic hydrogen need not be as large as required by Woods et al. (1982).

We acknowledge the support of NASA through grant NAGW-189.

\section{REFERENCES}

Bowman, W. C., Herbst, E., and De Lucia, F. C. 1982, J. Chem. Phys., 77, 4261

De Lucia, F. C. 1976, in Molecular Spectroscopy: Modern Research, ed. K. N. Rao (New York: Academic).

Gudeman, C. S., and Woods, R. C. 1982, Phys. Rev. Letters, 48 ,

Illies, A. J., Jarrold, M. F., and Bowers, M. T., private communication.

Light, J. C. 1967, Discuss. Faraday Soc., 44, 14.

Woods, R. C., et al. 1982, preprint. 1344.

Geoffrey A. BlaKe: Department of Chemistry, California Institute of Technology, Pasadena, CA 91125

Frank C. De Lucia and Eric Herbst: Department of Physics, Duke University, Durham, NC 27706

Paul Helminger: Department of Physics, University of South Alabama, Mobile, AL 36688 